\title{
Singular perturbation analysis of a mode initialization algorithm for simulating mode switching systems
}

\author{
Krister Edström \\ Department of Electrical Engineering \\ Linköping University, S-581 83 Linköping, Sweden \\ WWW: http://www.control.isy.liu.se \\ Email: edstrom@isy.liu.se
}

February 9, 1999

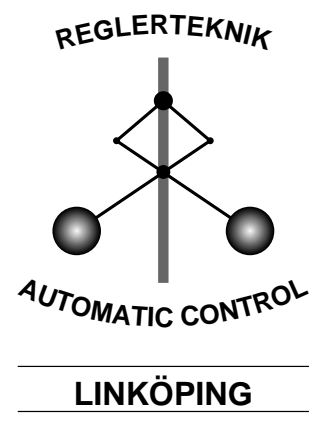

Report no.: LiTH-ISY-R-2047

Submitted to CDC '98

Technical reports from the Automatic Control group in Linköping are available by anonymous ftp at the address ftp.control.isy.liu.se. This report is contained in the compressed postscript file 2047.ps.Z. 


\title{
Singular perturbation analysis of a mode initialization algorithm for simulating mode switching systems
}

\author{
Krister Edström \\ Dept. of Electrical Engineering \\ Linköpings Universitet \\ SE-581 83 Linköping, Sweden \\ edstrom@isy.liu.se
}

\begin{abstract}
An initialization algorithm for the continuous states in mode switching systems is shown to give correct initial values. The mode switching systems are modeled with switched bond graphs, and the proof is based on singular perturbation theory.
\end{abstract}

\section{Introduction}

When simulating a physically modeled mode switching system [1], the state space may change size when changing modes. The problem of initializing the new mode becomes non-trivial. From a physical point of view, the initialization rules come from a generalization of the principle of momentum conservation $[5,2]$.

In this paper a physically based initialization algorithm for a system modeled with switched bond graphs [6] is analyzed using singular perturbation theory.

All proofs have been omitted and can be found in [3].

\section{A mode initialization algorithm}

Assume that the switched bond graph model has the following properties:

A1. There are no multi-port elements in the bond graph.

A2. There are no structural loops in the bond graph.

A3. There are no transformers or gyrators. This is to simplify notation. The algorithm can be extended to bond graphs with transformers and gyrators.

We also need the following definition:

\section{Definition 1 (Dependent set)}

In a bond graph with one storage element $s$ with nonpreferred causality, the dependent set $\mathfrak{D}$ consists of $s$ and all elements that have a direct causal path [7] leading to $s$.

When, during a simulation run, a transition between two modes, denoted $M_{1}$ and $M_{2}$, occurs the following assumptions are made about the transition and the mode being left, $M_{1}$.
A4. There are no causal conflicts in $M_{1}$.

A5. One switch changes states when changing modes.

These assumptions can be relaxed. They are introduced to simplify notation and proofs.

The initialization algorithm is the following:

\section{Algorithm 1 (Initialization algorithm)}

1. Save the value of the continuous state $x\left(t^{\prime}\right)$. $t^{\prime}$ denotes the time before the mode change and the time after the mode change. Since the mode change is instantaneous, $t-t^{\prime}<\delta$ for any choice of $\delta>0$.

2. Propagate causality in mode $M_{2}$.

3. Find $\mathfrak{D}_{2}$, the dependent set in $M_{2}$. Let $l_{1}$ and $l_{2}$ be the number of storage elements and sources respectively in $\mathfrak{D}_{2} . s_{1}$ is the element in $\mathfrak{D}_{2}$ with non-preferred causality, $s_{j}, j=2, \ldots, l_{1}$, the other storage elements in $\mathfrak{D}_{2}$, and $s_{j}, j=l_{1}+1, \ldots, n_{1}$, the storage elements in $\mathfrak{C D}_{2} . p_{j}, j=1, \ldots, l_{2}$, are the sources in $\mathfrak{D}_{2}$, and $p_{j}, j=l_{2}+1, \ldots, n_{2}$, the sources in $\mathfrak{C D}_{2}$.

Introduce a state variable $x_{j}$ for each storage element $s_{j}$ and an input variable $u_{j}$ for each source $p_{j}$.

4. Use a standard bond graph equation generation algorithm to derive an equation $x_{1}=g_{1}^{s}\left(x_{2}, \ldots, x_{l_{1}}\right)+$ $g_{1}^{p}\left(u_{1}, \ldots, u_{l_{2}}\right)$. Rewrite the equation as:

$$
x_{1}-g_{1}^{s}\left(x_{2}, \ldots, x_{l_{1}}\right)=g_{1}^{p}\left(u_{1}, \ldots, u_{l_{2}}\right)
$$

5. Derive $l_{1}-1$ equations $g_{2}, \ldots, g_{l_{1}}$, expressing $\dot{x}_{j}$, $j=2, \ldots, l_{1}$ in the rate variable $\dot{x}_{1}$, using the causal path between $s_{j}$ and $s_{1}$, and neglecting all other causal paths leading to $s_{j}$ :

$$
\dot{x}_{j}=g_{j}\left(\dot{x}_{1}\right)=\sigma_{j 1} \dot{x}_{1}, j=2, \ldots, l_{1}
$$

6. Rewrite and integrate both sides of $g_{j}, j=2, \ldots, l_{1}$, across the change of mode to achieve new equations:

$$
x_{j}(t)-\sigma_{j 1} x_{1}(t)=x_{j}\left(t^{\prime}\right)-\sigma_{j 1} x_{1}\left(t^{\prime}\right)
$$

7. All storage elements outside $\mathfrak{D}_{2}$ have preferred causality, therefore they will be continuous:

$$
x_{j}(t)=x_{j}\left(t^{\prime}\right), j=l_{1}+1, \ldots, n_{1}
$$

Equations (1), (3), and (4), form a system of equations that is solved to get the initial values of the new mode: 


\section{Lemma 1}

The system of equations generated in Algorithm 1 is solvable when assuming that Equation (1) is linear:

$$
\begin{aligned}
& g_{1}^{s}\left(x_{2}, \ldots, x_{l_{1}}\right)=\sigma_{12} a_{2} x_{2}+\ldots+\sigma_{1 l} a_{l_{1}} x_{l_{1}} \\
& g_{1}^{p}\left(u_{1}, \ldots, u_{l_{2}}\right)=b_{1} u_{1}+\ldots+b_{l_{2}} u_{l_{2}}
\end{aligned}
$$

where $a_{i} \geq 0$, and $\sigma_{1 i}$ is either +1 or $-1, i=2 \ldots l_{1} \diamond$

\section{Singular perturbation analysis}

We will here argue that the achieved values are correct by using the theory of singular perturbations [4]. Consider the instant change of state variable values as a very fast continuous change. Then there will be two time scales in the model; one fast describing the dynamics earlier modeled as instant changes, and one slow, describing the rest of the dynamics. By separating the two time scales, the slow dynamics can be considered constant while the fast dynamics reaches steady state. We will show that the steady state value of the fast dynamics and the constant value of the slow dynamics are the values achieved by Algorithm 1 in Section 2 .

Replace the switch that changes state with a linear R-element, $e=R \cdot f$, and assume that the constant $R$ changes value when the mode change appear. In the new model, the causality does not change when changing modes.

Here we will only consider $\mathbf{C}$-elements as storage elements. Therefore we will give the parameter $R$ in the added $\mathbf{R}$-element a small value $\epsilon$. The proofs for $\mathbf{I}-$ elements will be dual, if the parameter value of the added $\mathbf{R}$-element is chosen to be $R=1 / \epsilon$.

When generating equations from the new bond graph, we will get a state space description where the right hand side depends on $\epsilon$. Introduce new states $y=\left(y_{1}, \ldots, y_{n}\right)^{T}$, related to the old states $x=$ $\left(x_{1}, \ldots, x_{n}\right)^{T}$, with the following transformation:

$$
y=T x
$$

where the transformation matrix is derived from Equations (1), (3) and (4):

$$
T=\left[\begin{array}{ccccccc}
1 & -\sigma_{12} a_{2} & \ldots & -\sigma_{1 l_{1}} a_{l_{1}} & 0 & \ldots & 0 \\
-\sigma_{21} & 1 & \ldots & 0 & 0 & \ldots & 0 \\
\vdots & \vdots & \ddots & \vdots & \vdots & \ddots & \vdots \\
-\sigma_{l_{1} 1} & 0 & \ldots & 1 & 0 & \ldots & 0 \\
0 & 0 & \ldots & 0 & 1 & \ldots & 0 \\
\vdots & \vdots & \ddots & \vdots & \vdots & \ddots & \vdots \\
0 & 0 & \ldots & 0 & 0 & \ldots & 1
\end{array}\right]
$$

With this change of basis the following theorem holds:

\section{Theorem 1}

In the switch bond graph model, replace the switch that is being toggled at the mode change with a linear $R$ element with parameter value $\epsilon$. Derive the state space equations for the bond graph. Make a change of bases of the state space equation according to Equation (7).
The structure of the state space form will then be the following:

$$
\left[\begin{array}{c}
\epsilon \dot{y}_{1} \\
\dot{y}_{2: n}
\end{array}\right]=\left[\begin{array}{cc}
A_{1,1}+\epsilon A_{1,1}^{\epsilon} & \epsilon A_{1,2: n} \\
A_{2: n, 1} & A_{2: n, 2: n}
\end{array}\right]\left[\begin{array}{c}
y_{1} \\
y_{2: n}
\end{array}\right]+\left[\begin{array}{c}
B_{1}+\epsilon B_{1}^{\epsilon} \\
B_{2}
\end{array}\right] u
$$

where $A_{1,1} \neq 0$ and $B_{1} / A_{1,1} u$ equals the right hand side of Equation (1).

The fast dynamics correspond to $y_{1}$ and the slow dynamics to $y_{2: n}$. The time scale is separated by letting $\epsilon$ tend to zero. In the limit we get

$$
\begin{aligned}
y_{1} & =\frac{B_{1}}{A_{1,1}} u \\
\dot{y}_{2: n} & =A_{2: n, 2: n} y_{2: n}+B_{2} u
\end{aligned}
$$

By comparing Equation (10) with Equations (1), (5), and the first row in Equation (8) it is clear that Equations (10) and (1) are similar.

By assuming that the slow dynamics are constant, $y_{2: n}(t)=y_{2: n}\left(t^{\prime}\right)$, Equations (3), and (4) are achieved.

Hence the algorithm gives the same initial values as the ones achieved by introducing energy dissipation and letting the dissipation tend to zero.

\section{Conclusions}

For a very simple switched bond graph the algorithm gives the correct initial values, but it remains to extend the proofs to a larger class of bond graphs.

\section{References}

[1] K. Edström. Simulation of mode switching systems using switched bond graphs. Linköpings Universitet, December 1996. Lic. thesis No. 586.

[2] K. Edström. Mode initialization when simulating switched bond graphs. In Proc. of 2nd IMACS International Multiconference: CESA'98, Computational Engineering in Systems Applications. IMACS, April 1998.

[3] K. Edström. Singular perturbation analysis of a mode initialization algorithm for simulating mode switching system, long version. Technical report, Linköpings Universitet, 1998.

[4] P. Kokotović, H.K. Khalil, and J. O'Reilly. Singular perturbation methods in control: Analysis and design. Academic Press, 1986.

[5] P.J. Mosterman. Hybrid system dynamics: A hybrid bond graph modeling paradigm and its application in diagnosis. $\mathrm{PhD}$ thesis, Graduate School of Vanderbilt University, 1997.

[6] J.-E. Strömberg. A mode switching modelling philosophy. PhD thesis, Linköpings Universitet, 1994.

[7] J. van Dijk. On the role of bond graph causality in modelling mechatronic systems. $\mathrm{PhD}$ thesis, University of Twente, 1994. 